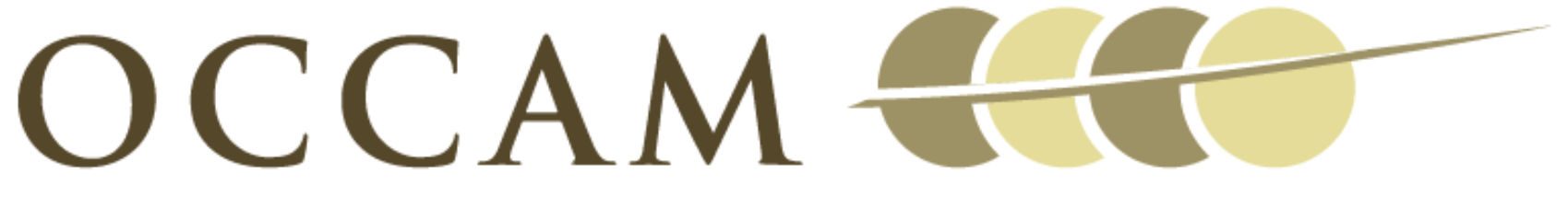

OXFORD CENTRE FOR COLLABORATIVE APPLIED MATHEMATICS

\author{
Report Number 10/01
}

Fluctuations and instability in sedimentation

\author{
by
}

Élisabeth Guazzelli, John Hinch

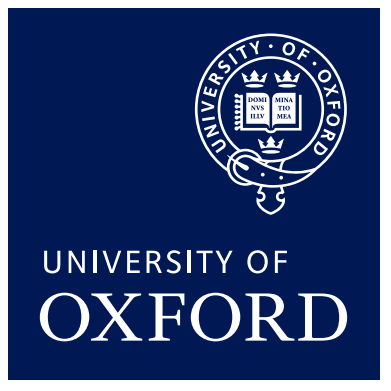

Oxford Centre for Collaborative Applied Mathematics Mathematical Institute 24 - 29 St Giles'

Oxford

OX1 3LB

England 



\title{
Fluctuations and instability in sedimentation
}

\author{
Élisabeth Guazzelli ${ }^{1}$ AND John HinCH ${ }^{2}$ \\ ${ }^{1}$ Institut Universitaire des Systèmes Thermiques Industriels, Centre National de \\ la Recherche Scientifique, Aix-Marseille Université, 13453 Marseille cedex 13; \\ email: Elisabeth.Guazzelli@polytech.univ-mrs.fr \\ ${ }^{2}$ Department of Applied Mathematics and Theoretical Physics, University of \\ Cambridge, Cambridge CB3 0WA; email: E.J.Hinch@damtp.cam.ac.uk
}
Key Words suspension, low Reynolds number, slender body, diffusion, struc- tural instability

\begin{abstract}
This review concentrates on the fluctuations of the velocities of sedimenting spheres, and on the structural instability of a suspension of settling fibers. For many years, theoretical estimates and numerical simulations predicted the fluctuations of the velocities of spheres to increase with the size of the container, whereas experiments found no such variation. Two ideas have helped our understanding. First, the correlation of the velocity fluctuations was found experimentally to be twenty interparticle separations. Second in dilute suspensions, a vertical variation in the concentration due to the spreading of the front with the clear fluid can inhibit the velocity fluctuations. In a very dilute regime, a homogeneous suspension of fibers suffers a spontaneous instability in which fast descending fiber-rich columns are separated by rising fiber-sparse columns. In a semi-dilute regime, the settling is hindered, more so than for spheres.
\end{abstract}

\section{CONTENTS}


FLUCTUATIONS IN SETTLING SPHERES . . . . . . . . . . . . . . . 3

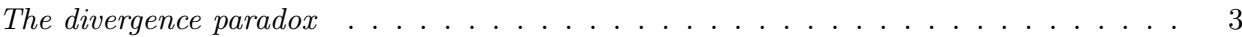

Velocity fluctuations and their correlation lengths . . . . . . . . . . . 8

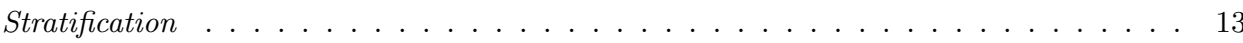

Hydrodynamic diffusion . . . . . . . . . . . . . . . . . . 17

INSTABILITY IN SETTLING FIBERS . . . . . . . . . . . . . . . . . 20

The observed regimes of sedimentation . . . . . . . . . . . . . . . . . 20

The structural instability . . . . . . . . . . . . . . . . . . . 24

Clusters and streamers . . . . . . . . . . . . . . . . . . . . 25

CONCLUDING REMARK AND FUTURE ISSUES . . . . . . . . . . . . . 29

\section{INTRODUCTION}

Sedimentation featured in an Annual Review 25 years ago by Davis \& Acrivos. That review considered the average settling velocity of spherical particles, which controls the rate of production of sediment and of pure liquid, see Supplemental Movie 1. For monodisperse suspensions of spheres, there had been recent theoretical calculations for the dilute limit, a difficult problem due to long range hydrodynamic interactions. There had also been experimental and theoretical progress on enhanced separation in inclined channels in the Boycott effect. Open questions were identified of whether the interparticle separation played a role in the dilute limit, and the extension to non-spherical particles. The review concluded with a remark that although the subject had a long history there were opportunities for future research, a remark well justified by the activity in the last 25 years. 
In this review we will concentrate on recent progress in understanding fluctuations during the sedimentation of spheres, and recent studies of instabilities of suspensions of sedimenting fibers.

\section{FLUCTUATIONS IN SETTLING SPHERES}

\subsection{The divergence paradox}

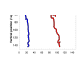

Figure 1: Tracking of two marked spheres in the midst of a $30 \%$ volume fraction sedimenting suspension of unmarked spheres made optically transparent by matching the index of refraction of the suspending fluid to that of the glass spheres (Nicolai et al. 1995): The particle trajectories are tortuous and exhibit large as well as small loops as the spheres sometimes moved upwards, against gravity.

Individual particle motions fluctuate about the mean, see Figure 1, due to the constantly changing configuration of the suspension microstructure and the 
resulting long-range multi-body hydrodynamic interactions. Consequently, even in a monodisperse suspension with no large differences in Stokes velocities among the particles, there exist distributions of velocities both parallel and perpendicular to gravity. The variance or the standard deviation of the velocity distribution is the simplest measure of the particle velocity fluctuations.

Until recently, there has been a paradox in which the variance in the velocities of sedimenting particles was predicted by theory and numerical simulations to depend on the size of the container, whereas experiments saw no such dependency. In this section, we describe the basis of this paradox.

At low Reynolds numbers, the hydrodynamic interactions between particles are long-ranged. The disturbance velocity at a distance $r$ caused by a sphere of radius $a$ sedimenting at $V_{S}$ is $O\left(V_{S} a / r\right)$ where the Stokes velocity is $V_{S}=\frac{2}{9} \frac{\left(\rho_{p}-\rho\right) a^{2} g}{\mu}$ ( $\rho_{p}$ is the density of the particle, $\rho$ that of the fluid, $\mu$ the viscosity of the fluid, and $g$ acceleration of gravity). This long-ranged interaction causes problems in theoretical calculations of averaged effects. For the dilute limit, a naive pairwise addition of the disturbance velocities from all the other spheres sedimenting in a spherical domain of radius $R$ would yield a change in the sedimentation velocity of a test sphere of

$$
O\left(\int^{R} V_{S} \frac{a}{r} n d V\right)=O\left(V_{S} \phi\left(\frac{R}{a}\right)^{2}\right)
$$

where $n$ is the number density of particles in the suspension and $\phi=n \frac{4 \pi}{3} a^{3}$ is their volume fraction. This theoretical estimate suggests that the mean settling velocity of the particles is not an intrinsic property of the suspension, but depends on the size, and possibly the shape, of the container. Experimental observations, however, had clearly established the hindered settling result $\langle w\rangle=V_{S}(1-k \phi)$, for the mean settling velocity of particles in the dilute limit $\phi \ll 1$, with $k$ a constant 
$\approx 5$. This is the dilute limit of the Richardson-Zaki law $\langle w\rangle=V_{S}(1-\phi)^{n}$ with $n \approx 5.1$ at low Reynolds numbers.

The paradox for the mean settling velocity was resolved by Batchelor (1972) who noted that there was a multi-particle effect of a back-flow of $-5.5 V_{S} \phi$ at locations where the test sphere could be placed. He split the hydrodynamic interaction into a contribution to the back-flow which gave a divergent integral and a remainder which gave a convergent integral. This resulted in his estimate $k=6.55$ for a uniform distribution of the separation of pairs of particles. An alternative analysis was given by Hinch (1977) in which the leading order $V_{S} a / r$ interaction was seen as that of a point force which could instead be considered as changing the density from that of the suspending liquid to that of the suspension.

Turning now to a calculation of the variance of the fluctuating velocities of the settling particles, a similar naive pairwise addition results in an estimate

$$
\left\langle w^{\prime 2}\right\rangle=O\left(V_{S}^{2} \phi \frac{R}{a}\right)
$$

(Caflisch \& Luke 1985, Tory \& Pickard 1986). The question therefore arises again of whether the variance does truly depend on the size of the container or whether it is an intrinsic property which must be calculated by another method.

Hinch (1988) answered this question by considering a blob of size $\ell$. This would contain on average $n \ell^{3}$ particles, but there would be statistical fluctuations of $\sqrt{n \ell^{3}}$ in the number if the particles were positioned randomly and independently. This fluctuation in the number gives a fluctuation in the weight of the blob of $m g \sqrt{n \ell^{3}}$, where $m$ is the mass of a particle compensated for buoyancy. Balancing the fluctuation in weight with a Stokes drag on the blob of size $\ell$ yields a 
fluctuation in velocity of

$$
w^{\prime}=O\left(\frac{m g \sqrt{n \ell^{3}}}{6 \pi \mu \ell}\right)=O\left(V_{S} \sqrt{\phi \frac{\ell}{a}}\right) .
$$

As larger blobs give larger fluctuations in velocity, one would expect to see those corresponding to the largest spherical blob which can be fitted into the container, i.e. limited by the smallest of the height, width and depth, say $L$.

The above theoretical estimate is supported by numerical simulations. When particles are positioned randomly and uniformly throughout the container, then the initial value of the variance is found to be proportional to the size of the container, Koch (1994) and Cunha et al. (2002) summing the interaction between point-particles, Ladd (1996 and 1997) using a Lattice Boltzmann method, and Bergougnoux et al. (2003) and Mucha et al. (2004) using a truncated Fourier series for point-particles. However, experiments have found that the steady state value of the variance does not depend on the size of the container (Nicolai \& Guazzelli 1995), see Section 2.2. The paradox for the variance in the velocities of sedimenting particles is therefore that theory and simulations contradict experiments.

This clear disagreement between theory and experiments has become less clear cut in time with the arrival of new observations which will attempt to explain in Sections 2.2 and 2.3. Segrè et al. (1997) found a dependence on the size of container if the container was small, which Brenner (1999) showed followed the theoretical estimate (1). The simulations of Bergougnoux et al. (2003), Mucha et al. (2004), Nguyen \& Ladd (2005) found that the velocity fluctuations decay in time from the theoretical estimate. The experiments of Guazzelli (2001), later confirmed by Chehata Gómez et al. (2009), found that the initial value of the fluctuations does depend on the size of the container and does follow the theoret- 
ical estimate. The paradox has now moved to what determines the well defined steady value of the experimental fluctuations after the decay from the initial value, this steady value normally being independent of the size of the container.

Several modifications of the theory have been suggested aimed at making predictions that do not depend of the size of the container. Koch \& Shaqfeh (1991) noted that the long-range hydrodynamic interactions would be screened if each particle was surrounded by sufficient buoyant clear fluid so that the net weight of the volume was precisely equal to that of the suspension in the same volume. However, Ladd (1996) found that there was insufficient volume of clear fluid to make the particle appear neutrally buoyant. Moreover, the volume of clear fluid would have to extend to the interparticle separation $a \phi^{-1 / 3}$, which would make the suspension look more like a random crystal.

The estimate of the variance in the velocity fluctuations uses the Stokes drag law applied to the blob. While a Reynolds number $R e_{p}=V_{S} a / \nu$ based on the radius $a$ is mostly very small in the experiments, a Reynolds number based on the size of the container $R e=R e_{p} L / a$ may not be small. An estimate of the variance for this case was given by Hinch (1988), $\left\langle w^{\prime 2}\right\rangle=O\left(V_{S}^{2} \phi^{2 / 3} R e_{p}^{-2 / 3}\right)$, which is independent of the size of the container. Koch (1993) and Yin \& Koch (2008) have looked at the case of the particle Reynolds number taking moderate values.

A theoretical suggestion by Luke (2000) and taken up by Tee et al. (2002) is that a small vertical variation in the concentration of the particles can lead to a density stratification which can inhibit the velocity fluctuations. We will return to this important mechanism in Section 2.3

Finally we should mention two further ideas. The topic of fluctuations was 
reviewed from a statistical mechanics perspective by Ramaswamy (2001), using theoretical ideas from Levine et al. (1998). Randomness was characterized in the standard way by a strong diffusion process with white-noise forcing, with sedimentation as a minor linearized perturbation, which it is not. In contrast, Tong \& Ackerson (1998) considered particle diffusion small and sedimentation dominant, and drew analogies with turbulent thermal convection. Small structures created next to the bottom heated plate have a size $R a^{-1 / 3}$ where $R a$ is the Rayleigh number, which translates through the analogy into $11 a \phi^{-1 / 3}$; pity there is no analogous heated plate in sedimentation.

\subsection{Velocity fluctuations and their correlation lengths}

The variability of the velocities was recognized in early experiments in the 70 s, see Tory et al. 1992. Later, large velocity fluctuations were measured by tracking marked spheres in an otherwise transparent sedimenting suspension (Ham \& Homsy 1988, Nicolai et al. 1995), see Figure 1. The measurements were not collected immediately after the initial mixing of the suspension but the suspension was allowed to settle for some time and reach a steady behavior before tracking started. They were not seen to be affected by the size of the container in the large containers used (Nicolai \& Guazzelli 1995).

Using Particle Image Velocimetry (PIV), Segrè et al. (1997) measured the velocity field of the particles, from which they could construct the spatial correlation function. They found that the horizontal length over which the vertical velocities were correlated was around twenty interparticle separation, $20 a \phi^{-1 / 3}$. This value of the correlation length seems to be a robust result for volume fractions $\phi$ ranging from $10^{-4}$ to 0.4 (Segrè et al. 1997, Segrè et al. 2001, Guazzelli 2001, Snabre 

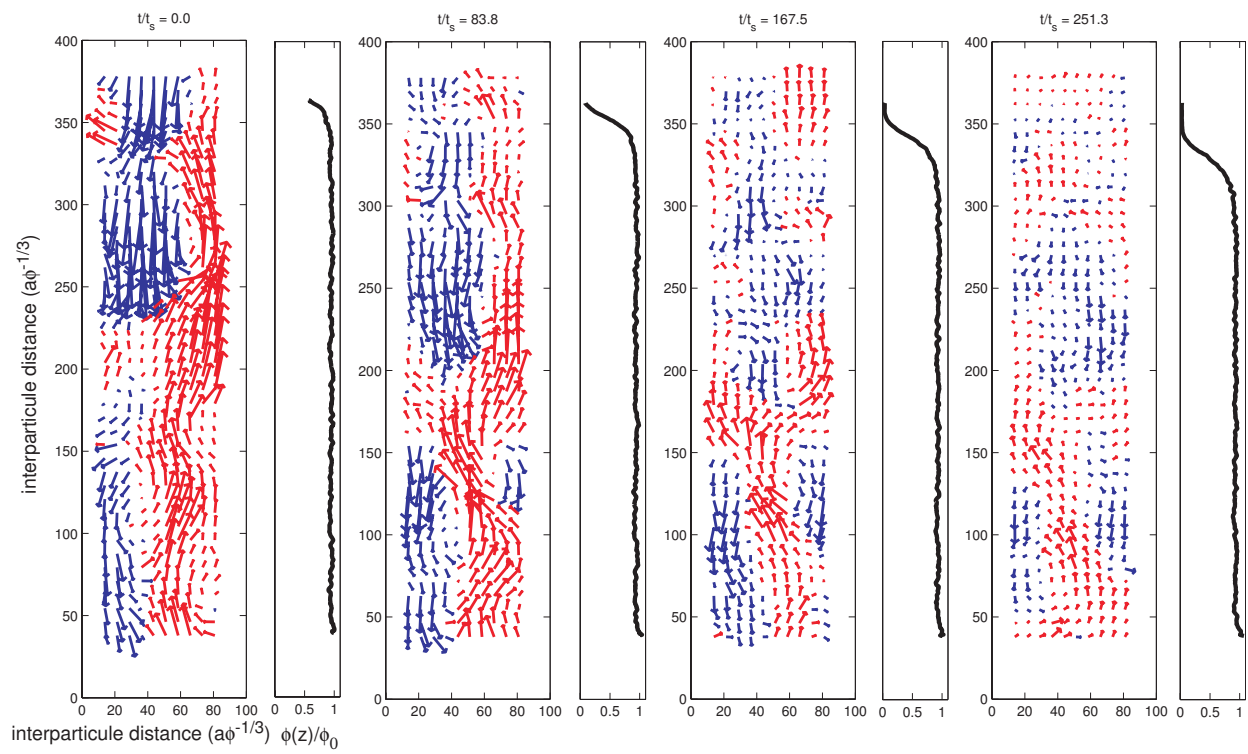

Figure 2: Relaxation of the large-scale fluctuations: Velocity field coming from Particle Image Velocimetry (PIV) sampling the whole cell height and width within a laser sheet located in the middle plane and concentration profile coming from light-attenuation measurement through the suspension (Bergougnoux et al. 2003, Chehata Gómez et al. 2009). The time scale is the Stokes time $t_{S}=a / V_{S}$, i.e. the time for an isolated sphere to sediment its radius.

et al. 2009). This correlation length plays a central role in understanding the velocity fluctuations.

When the minimum dimension of the container $L$ was less than $20 a \phi^{-1 / 3}$, Segrè et al. (1997) found that the velocity fluctuations depended on the size of the container and follow the theoretical estimate (1) with the size of the blob $\ell=L$. When $L>20 a \phi^{-1 / 3}$, they found the steady vertical velocity fluctuations were $2 V_{S} \phi^{1 / 3}$ independent of $L$, which corresponds to (1) with $\ell=20 a \phi^{-1 / 3}$.

These experimental results were confirmed by numerical simulations by Nguyen $\&$ Ladd (2004) using a Lattice Boltzmann method at $\phi=0.13$ and $R e_{p}=0.1$. 

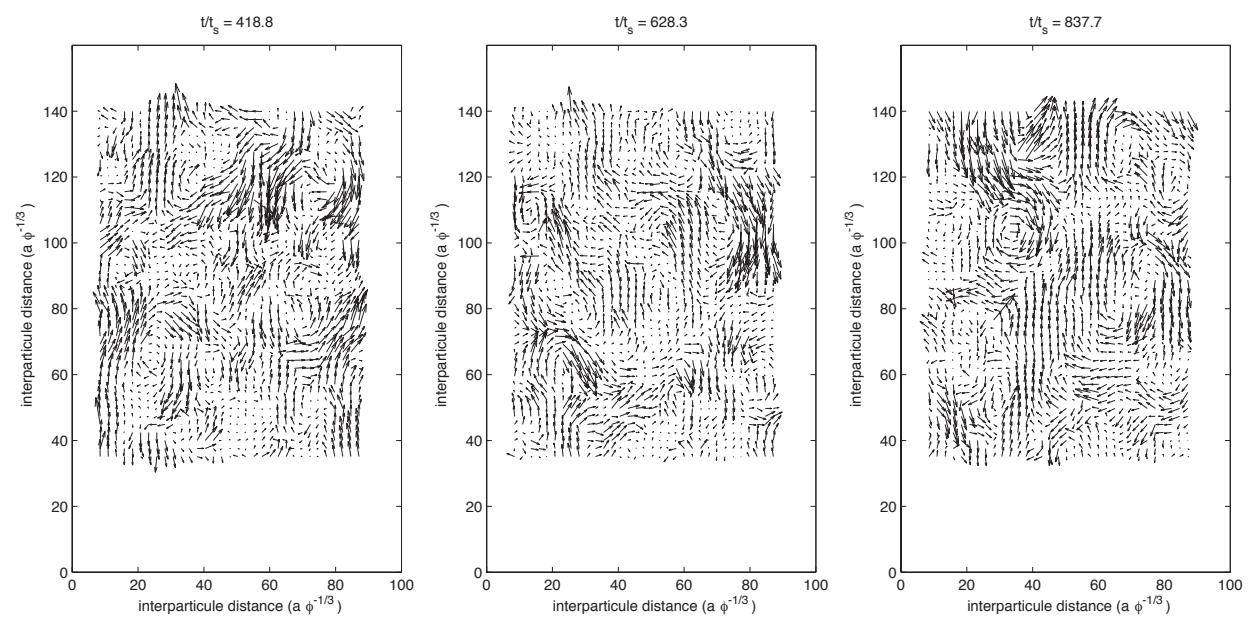

Figure 3: Dominance of the remaining smaller-scale fluctuations until the arrival of the upper sedimentation front: Velocity field coming from Particle Image Velocimetry (PIV) sampling the entire cell cross-section for a window covering the lower 1/4 of the cell height (Bergougnoux et al. 2003, Chehata Gómez et al. 2009). The time scale is the Stokes time $t_{S}=a / V_{S}$.

With periodic boundary conditions, the velocity fluctuations were constant in time at a value given by (1) with $\ell=L$. However if impenetrable top and bottom boundaries were used, the velocity fluctuations decayed in time to a steady value which depended on the size of the container $L$ if $L<20 a \phi^{1 / 3}$, and was independent if $L>20 a \phi^{-1 / 3}$. The importance of impenetrable top and bottom boundaries had been found earlier by Ladd (2002) and Cunha et al. (2002), taking up the speculation of Hinch (1988) that convection currents would remove long wavelength horizontal density fluctuations leaving the irreducible scale of the interparticle separation.

While the steady state velocity fluctuations are independent of the size of the container if $L>20 a \phi^{-1 / 3}$, the early fluctuations do depend on the container. Guazzelli (2001) saw large-scale fluctuations of the size of the width of 
Figure 4: Velocity fluctuations normalized by the mean $\langle w\rangle$ in the (left in blue) vertical and (right in red) horizontal directions versus volume fraction. The darkblue line is the correlation $3 \phi^{1 / 3}$ and the light-blue line $2 \phi^{1 / 3}$. The dark-red line is the correlation $1.5 \phi^{1 / 3}$ and the light-red line $\phi^{1 / 3}$. Experiments: open diamonds at $R e_{p}<10^{-4}$ (Ham \& Homsy 1988), filled circles at $R e_{p}<10^{-3}$ (Nicolai et al. 1995), light-color filled diamond at $R e_{p} \approx 10^{-4}$ (Segrè et al. 1997), dark-color filled diamond at $R e_{p} \approx 10^{-4}$ (Segrè et al. 2001), dark-color filled down-triangle at $R e_{p}<10^{-4}$ (Chehata Gómez et al. 2009), light-color filled up-triangle at $R e_{p} \approx 10^{-3}$ (Snabre et al. 2009). Inset: Linear plot of relative fluctuations versus volume fraction.

the container which dominated the dynamics just after the initial mixing of the suspension, see Figure 2. The initial magnitude of the velocities follow (1) with $\ell=L$ (Chehata Gómez et al. 2009). Moreover they are strongly anisotropic with vertical velocities around four times the horizontal. Figure 2 shows the initial strong large-scale fluctuations decay in time to weaker small-scale fluctuations. These small-scale fluctuations remain in a steady state until the sedimentation front arrives in the imaging window, see Figure 3. This reduction of the initially 
large fluctuations to a smaller steady value independent of the size of the container seen initially at low volume fractions $\phi$ has been also observed at larger $\phi$ (Snabre et al. 2009). This behavior is consistent with the above speculation in which heavy blobs fall to the bottom and light rise to the top.

Figure 4 collects most of the available experimental data for the velocity fluctuations in the steady regime obtained both from Particle Tracking (PT) and Particle Image Velocimetry (PIV). The velocity fluctuations increase roughly as $\phi^{1 / 3}$ at low $\phi$, although there is some uncertainty in the coefficient. The vertical fluctuations reach a maximum around $\phi=0.3$, where they are 1.7 times the mean settling speed, and then decrease. The anisotropy between the vertical and horizontal fluctuations, which was four initially, drops to two in the steady state and is even smaller for $\phi>0.2$.

Similar large velocity fluctuations to those for $0.2<\phi<0.4$ have been seen in fluidized beds at low Reynolds number, e.g. by multiple light-scattering (Xue et al. 1992) and by ultrasonic correlation spectroscopy (Cowan et al. 2000). However note that, although it is often said that a fluidized bed is equivalent to steady sedimentation, the superficial velocity driving the flow in a fluidized bed vanishes at the walls and this leads to a convection motion of the particles at low Reynolds numbers.

While the microstructure of the suspension plays an important role in the behavior, there have been relatively few detailed studies, which often contradict one another, as discussed in Section 2.1 (Koch \& Shaqfeh 1991, Ladd 1996). Experiments by Lei et al. (2001) found developing in time that large regions had smaller fluctuations in the number of particles than expected for independently randomly positioned particles. Mucha et al. (2004) saw a similar shortfall in 
numerical simulations. On the other hand, Bergougnoux \& Guazzelli (2009) found in experiments large regions had larger fluctuations than expected, which did not change in time. They also observed small regions devoid of particles, and so suggest that the role of the initial mixing on the microstructure should be explored. The structure function $S(\mathbf{k})$ is difficult to access experimentally for non-Brownian particles. In numerical simulations Nguyen \& Ladd (2005) found in the steady state that the horizontal fluctuations in density were suppressed.

\subsection{Stratification}

The possibility that an unnoticed vertical variation in concentration of the particles might influence experiments was first drawn to our attention by Bławdziewicz circa 1995. However, the robustness of our experimental results (Nicolai \& Guazzelli 1995, Nicolai et al. 1995) to such an uncontrolled variation lead us to dismiss the good suggestion. Later Luke (2000) showed how the velocity fluctuations would decay in time in a stably stratified suspension as the density fluctuations moved to their neutral buoyancy level. For that calculation, it was not clear what would create the stratification.

In 2002, Tee et al. made two important observations. First, they suggested that the stratification came from the spreading of the interface between the suspension and the clear fluid above. In very dilute suspensions, the interface is diffuse because the self-sharpening caused by hindered settling is negligible. Second, they estimated the small stratification required to influence the velocity fluctuations. For a cube of size $L$ equal to the minimum dimension of the container, they argued stratification would be important when the variation in the number of particles in the cube due to stratification $L \partial\left(n L^{3}\right) / \partial z$ exceeded the statistical 
fluctuations $\sqrt{n L^{3}}$, i.e. when a nondimensional measure of the stratification $\beta$ exceeded a critical value

$$
\beta=-\frac{a}{\phi} \frac{\partial \phi}{\partial z}>\beta_{c}=\phi^{-1 / 2}(L / a)^{-5 / 2}
$$

When the stratification exceeds this very small critical value, the largest blob with statistical fluctuations greater than those from stratification has size

$$
\ell=L\left(\beta_{c} / \beta\right)^{2 / 5}
$$

which gives a variance in the velocity fluctuations

$$
\left\langle w^{\prime 2}\right\rangle=O\left(V_{S}^{2} \phi \frac{L}{a}\left(\frac{\beta_{c}}{\beta}\right)^{2 / 5}\right) .
$$

This is independent of the size of the container $L$. These dominant fluctuations produce a hydrodynamic dispersion with a diffusivity

$$
D=w^{\prime} \ell=k V_{S} a \phi^{4 / 5}\left(-a \frac{\partial \phi}{\partial z}\right)^{-3 / 5},
$$

with $k$ a numerical constant.

In a subsequent paper (Mucha \& Brenner 2003), numerical simulations were made under different conditions. A local instantaneous diffusivity $D$ was evaluated as the flux of concentration divided by the concentration gradient

$$
D(z, t)=-\int_{z}^{\infty} \frac{\partial \phi}{\partial t} d z / \frac{\partial \phi}{\partial z}
$$

The diffusivity was found to be not a function of just the concentration $\phi$, but collapsed onto a single curve with the above dependence on $\phi^{4 / 5}(\partial \phi / \partial z)^{-3 / 5}$, with $k \approx 1$.

The diffusion equation with this nonlinear diffusivity has a similarity solution for the spreading front, with the thickness of the front growing in time as

$$
\delta=a \phi^{1 / 7}\left(V_{S} t / a\right)^{5 / 7}
$$


This prediction was tested in experiments by Bergougnoux et al. (2003), who found the thickness grew linearly in time, mostly due to unavoidable small polydispersity in the size of the spherical particles. Numerical simulations by Bergougnoux et al. (2003) with strictly monodisperse particles could not distinguish between the $t^{5 / 7}$ power-law growth and a linear growth in time within the range of data available. Numerical simulations in a later paper (Chehata Gómez et al. 2008) favored the linear growth, and experiments in the same paper confirmed the linear growth and found how it depended in detail on the polydispersity. It was thought possible that a linear growth might result from heavy blobs falling out the front leaving it depleted. That said, a universal form was found in both the experiments and the simulations for the concentration profile in the front, reminiscent of the unpublished similarity solution of the diffusion equation with the nonlinear diffusivity.

The velocity fluctuations in the spreading front were studied in numerical simulations by Mucha et al. (2004). They found where the stratification was very weak, $\beta<\beta_{c}$, the variance was proportional to the size of the container. However where the stratification exceeded the critical value, the variance was reduced by the factor $\left(\beta_{c} / \beta\right)^{2 / 5}$ given above, although there was considerable scatter in their $\log -\log$ plot.

In order to give a cleaner test of the effect of stratification, Tee et al. (2007) set up an experiment and numerical simulations with a controlled uniform stratification in the initial suspension. They found much clearer evidence of the $\left(\beta_{c} / \beta\right)^{2 / 5}$ reduction of the variance of the velocity fluctuations. The experimental variance was curiously smaller by a factor of 4 , corresponding the stratifications 32 times larger. This difference in now thought to be due to a gradient in sucrose con- 
tributing more to the density stratification than the very dilute concentration of particles.

It seems that the velocity fluctuations are effected by very small vertical gradients in concentration. The small gradients were measured in experiments for the first time by Chehata Gómez et al. (2007) in two illustrative experiments. In one experiment, the weak stratification seemed to control the velocity fluctuations before the arrival of the front. However in a second experiment, the velocity fluctuations were unaltered while the stratification was negative within the observation window, and so if stratification was influencing the velocities it was not an instantaneous local relation.

The current state of play comes from the more extensive experiments conducted by Chehata Gómez et al. (2009), who also made two sets of numerical simulations, one starting from an initially stratified suspension and the other from a uniform one. Both experiments and simulations found the initial velocity fluctuations followed the original Caflisch \& Luke scaling (1) with $\ell=L$. The fluctuations then decayed to a plateau value. In most of the experiments, the plateau value was not related to the local stratification. In the simulations with an initial stratification, the velocity fluctuations decayed by the expected factor of $\left(\beta_{c} / \beta\right)^{1 / 5}$. In the simulations starting from with a uniform suspension, the velocity fluctuations did not decay until the front arrived. Within the front, the fluctuations were reduced by the stratification factor $\left(\beta_{c} / \beta\right)^{1 / 5}$ in both experiments and simulations. The data from the simulations was less convincing, possibly due to a delay for the fluctuations to adapt to the changing conditions, and possibly due to the concentration gradient not being constant over the size of the important blobs. 
For the level of fluctuations in the plateau before the front arrives, the issue today seems to focus on what is the result of mixing in the experiments. Starting from a concentrated suspension, it is difficult to produce a homogeneous suspension, especially when it is dilute, while avoiding all entrainment of air by the mixer. One would be interested in the initial microstructure, density fluctuations, and possible mean stratification.

\subsection{Hydrodynamic diffusion}

We finally turn to the problem of hydrodynamic diffusion which was at the origin of the research on fluctuating motion of the particles because of it importance in mixing, although in recent times the topic has been much neglected. This diffusion process has been coined hydrodynamic diffusion as its origin lies in the hydrodynamic interactions between the particles (Davis 1996). Ham \& Homsy (1988) were the first to investigate the nature of the long-time motions of individual spheres in the midst of a sedimenting suspension of like spheres and to relate it to a Fickian diffusion process. Their findings were confirmed and extended by Nicolai et al. (1995), see Figure 1.

After a large number of hydrodynamic interactions, individual particles loose memory of the details of these interactions and undergo a random walk. The long time diffusive behavior of the fluctuating particle motions was demonstrated by examining an exponential relaxation of the particle velocity autocorrelation functions and the linear growth of the second-order moments of the particle displacements. Interestingly, the measured correlation times were found to be independent of the concentration with vertical times slightly longer than horizontal times, over a wide range of volume fraction from 0.05 to 0.4 . Self-diffusivities nor- 
Figure 5: Self-diffusivity [filled diamonds at $R e_{p}<10^{-4}$ (Ham \& Homsy 1988) and filled circles at $R e_{p}<10^{-3}$ (Nicolai et al. 1995)] in the vertical $D_{v}^{s}$ (blue) and horizontal $D_{h}^{s}$ (red) directions, normalized by the product of particle radius $a$ and mean velocity $\langle w\rangle$ versus volume fraction.

malized on the measured mean vertical velocity are constant over a substantial range of concentration from 0.05 to 0.3 , and display a strong anisotropy between the vertical and horizontal directions, $D_{v}^{s} \approx 8 a\langle w\rangle \approx 4 D_{h}^{s}$, as shown in Figure 5 . Again hydrodynamic self-diffusivities were seen not to be affected by the size of the container in these experiments collected in the steady state of sedimentation for large containers (Nicolai \& Guazzelli 1995). Above a volume fraction of 0.3, there is clearly a different regime with a strong decrease of the self-diffusivities and anisotropy. The basic mechanism for diffusion may be the fluctuating motion of clusters instead of the fluctuating motion of single particles, a feature that has been observed at high concentrations in numerical simulations (Ladd 1993). This higher concentration regime deserves more attention. 
There are only a limited number of numerical simulations that have attempted to address hydrodynamic diffusion (Ladd 1993, Ladd 1996, Koch 1994, Cunha et al. 2002). This is probably because the predicted divergence of the diffusivity is more severe $\left(D \sim w^{\prime 2} \ell \sim \ell^{3 / 2}\right)$ than that of the velocity fluctuations $\left(w^{\prime} \sim \ell^{1 / 2}\right)$ and the latter has received most attention; it would be interesting to return to the study of hydrodynamic diffusion. Simulations of hydrodynamic diffusion (and to a lesser extend velocity fluctuations) are not only affected by the size of the box but also its shape. Early dynamic simulations, which include the full hydrodynamic interaction between the particles using a multipole expansion, found an unrealistically large anisotropy in fluctuations, correlation time, and diffusivity (Ladd 1993). This was later attributed to the periodic boundaries imposed in the vertical direction of the cubic box, and was found to be remedied by increasing the aspect ratio of the box (Koch 1994). More realistic anisotropy has been found in lattice-Boltzmann simulations using an elongated box in the vertical direction (Ladd 1996) or dynamic simulations having an impenetrable lower boundary but still side periodicity (Cunha et al. 2002).

The self-diffusion that we have just discussed refers to the tracer diffusion of a test particle in the interior of a suspension where the particle concentration is uniform. But there is another diffusion phenomenon caused by concentration gradients which acts on a macroscopic scale. This so-called gradient diffusion refers to the drift of particles down a concentration gradient as a result of their fluctuating motion. The broadening of the interface between the sedimenting suspension and the clear fluid above has been attributed partly to this gradient diffusion. The spreading of this front has been measured with different techniques such as light attenuation and magnetic resonance imaging (Davis \& Hassen 1988, 
Lee et al. 1992, Turney, Cheung, McCarthy \& Powell 1995). However, other effects also control the front: polydispersity which also leads to broadening of the front but linearly in sedimentation time, hindered settling which reduces the spreading by a self sharpening process, and heavy blobs falling out of the front leaving it depleted. It is thus difficult to deconvolve the simultaneous actions of these effects and to identify the sole effect of gradient diffusion. In particular, even a small polydispersity (causing the front to grow linearly in time) rapidly overcomes the effect of diffusion (causing the front to grow diffusively with the square root of time if the diffusivity is constant). It may make sense then to analyze the early stage of sedimentation if one wants to determine the diffusion coefficient (Lee et al. 1992). When self-sharpening is accounted for (Lee et al. 1992, Martin et al. 1994), the gradient diffusivities are found to be $D^{g} \approx 20 a\langle w\rangle$ for concentration ranging from 0.02 to 0.1 (thus larger than self-diffusivities within the same range) while one finds apparent diffusivities $D_{a p p}^{g} \approx 8 a\langle w\rangle$ if the selfsharpening is ignored (Davis \& Hassen 1988, Turney, Cheung, McCarthy \& Powell 1995). However, these calculations should perhaps now be revisited in the light of the ideas emerging in Section 2.3.

\section{INSTABILITY IN SETTLING FIBERS}

\subsection{The observed regimes of sedimentation}

The case of non-spherical particles has received much less attention than that of spheres. The fundamental difference is that anisotropic particles orient in flow and drift perpendicular to gravity. Most of the recent focus has been on the sedimentation of suspensions of rod-like particles or fibers. The sedimentation of disk-like particles or platelets needs further study. The interest in the sedi- 
mentation of fibers lies in part in the availability of methods for slender bodies as well as in its importance in industrial applications such as the fabrication of fiber-reinforced materials and of pulp and paper.

Different regimes can be identified for suspensions of fibers of aspect ratio $A=l / d$ of length $l$ and diameter $d$. In the dilute regime, $n l^{3}<1$ where $n$ is the number density of particles, each fiber can rotate freely in the large volume $l^{3}$. The semi-dilute regime is reached for $n l^{3}>1>n l^{2} d$. There is a nematic phase transition in which the fibers become aligned at a concentration of $n l^{2} d \approx 1$, which may correspond to a small volume fraction, $\phi=\pi n l d^{2} / 4$ if the aspect ratio of the fiber $A$ is large. Most of the theoretical and numerical studies have been restricted to the dilute regime while both dilute and semi-dilute regimes have been examined experimentally.

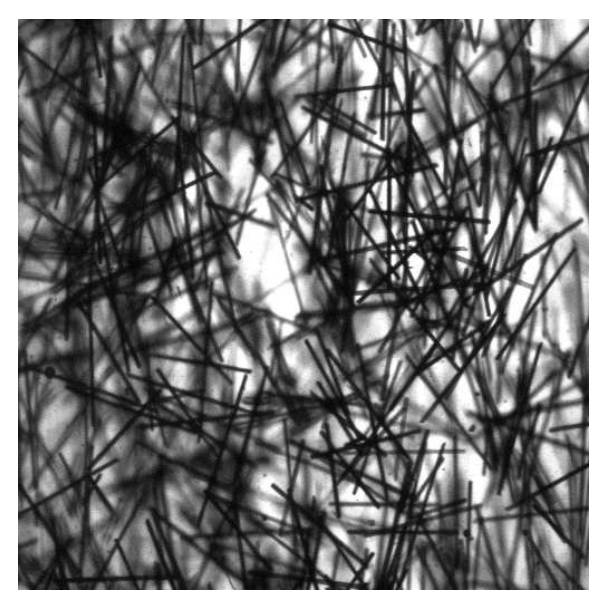

Figure 6: Photograph of an inhomogeneous sedimenting suspension exhibiting packets of fibers for $A=32$ and $\phi=0.5 \%$ (Herzhaft \& Guazzelli 1999).

Early experiments on dilute suspensions reported the formation of floc-like inhomogeneities settling at large velocities (Kumar \& Ramarao 1991). As the concentration was increased, the number of flocs increased and hindrance occurred. 
At higher concentrations, a semi-rigid network of fibers formed and the settling speed dropped abruptly. Hindered settling was observed in the semi-dilute regime using magnetic resonance imaging to follow the diffuse interface between between the clear fluid and the suspension, but no information was provided concerning the structure of the suspension (Turney, Cheung, Powell \& McCarthy 1995). By measuring the velocity of individual fibers in the dilute regime and the interface speed using optical techniques in the semi-dilute regime, Anselmet (1989) also reported hindered settling. However, her data are much lower than those of Turney et al. (1995), this discrepancy being attributed to the small size of her settling vessel. Later, fiber trajectories and orientations were examined in both the dilute and semi-dilute regimes (Herzhaft et al. 1996, Herzhaft \& Guazzelli 1999) by adapting the Particle Tracking technique used in the sedimentation of spheres. For all concentrations and aspect ratios studied, the fiber trajectories were found to have large anisotropic fluctuations and a strong alignment in the direction of gravity with occasional flippings. A steady state for the mean settling velocity and the orientation was always achieved.

These experiments identify two distinct regimes. In the dilute regime, visualization of the suspension shows that the fibers clump and form packets separated by sparse regions, see Figure 6 and Supplemental Movie 2. The large velocity gradients between the fast-settling packets and the sparse regions of backflow are considered to be the cause of the flipping motions. In this regime, the mean settling velocity is not hindered and can be larger than the Stokes velocity of an isolated vertical fiber $V_{S \|}$, as evidenced in Figure 7 (left). The mean velocity is found to increase at low $\phi$, to reach a maximum (more or less pronounced depending on $A$ ) at $\phi \approx 0.005$, and then to decrease with increasing $\phi$. No simple scaling 
Figure 7: Mean vertical velocity (left in blue) normalized by the Stokes velocity of a vertical fiber $V_{S \|}$ computed with using Equation (2.1) of Herzhaft \& Guazzelli (2007) and velocity fluctuations normalized by the mean in the vertical (right in blue) and horizontal (right in red) directions plotted as a function of fiber volume fraction. The solid black line is the Richardson-Zaki correlation $(1-\phi)^{n}$ with $n=9$. Experiments: $A=5$ (filled down-triangles), $A=11$ (filled squares), $A=20$ (filled diamonds), $A=32$ (filled circles) at $R e_{p} \approx 10^{-4}$ (Herzhaft \& Guazzelli 1999), $A=17$ (filled up-triangles) at $R e_{p} \approx 10^{-6}$ (Turney, Cheung, Powell \& McCarthy 1995), and $A=10$ (crosses) at $R e_{p} \approx 10^{-4}$ (Anselmet 1989). Simulations: $A=15.6$ (open up-triangles) (Mackaplow \& Shaqfeh 1998), $A=11$ (open squares) and $A=32$ (open circles) (Butler \& Shaqfeh 2002). Inset: Blowup of relative velocity fluctuations for low volume fractions.

has been found to describe this behavior, which is probably a complex function of both $\phi$ and $A$. For $\phi>0.01$, the fibers are still oriented in the direction of gravity, but the mean velocity becomes hindered and depends not on $n l^{3}$ but on the volume fraction $\phi$. The hindrance is more severe than in the case of spheres. 
A Richardson-Zaki law, $(1-\phi)^{n}$ with $n \approx 9$, is in very good agreement with data having different $A$ coming from both fiber-tracking (Herzhaft \& Guazzelli 1999) and interface measurements (Turney, Cheung, Powell \& McCarthy 1995), see Figure 7 (left). In this regime, visualization shows that the fibers form a loosely connected network and the fluid backflow goes up through holes in the network. In figure 7 (left), we also report the predictions of a Monte Carlo simulation using a slender-body approximation (Mackaplow \& Shaqfeh 1998) which are of the same order of magnitude as the experimental data. But the comparison should be handled with care since this simulation assumes that the suspension is homogeneous and the orientation isotropic.

The particle velocity fluctuations were found to increase with increasing $\phi$ across both dilute and semi-dilute regimes, as shown in Figure 7 (right). These fluctuations are seen to be very strong, reaching values of $\approx 9$ times in the vertical direction for $\phi=0.15$, see Figure 7 (right). The fluctuation anisotropy is independent of $\phi$ and $A$ and has a value $\approx 3$, larger than in the case of spheres. Another important finding is that the orientation distributions, which have a substantial alignment in the direction of gravity, are quite similar whatever $\phi$ and $A$.

\subsection{The structural instability}

A linear stability analysis has predicted the instability observed at low concentration (Koch \& Shaqfeh 1989). They consider alternating tall columns with more and less fibers as the number density of fibers varies horizontally as $n_{0}+$ $n_{1}(t) \cos k x$. At low Reynolds numbers, this variation in number density drives a 
vertical flow

$$
w_{1}=-\frac{n_{1} m g}{\mu k^{2}} \cos k x
$$

where $m g$ is the weight of a fiber adjusted for buoyancy. The shear $\partial w_{1} / \partial x$ will start to rotate the fibers as

$$
\frac{\partial \theta_{1}}{\partial t}=\frac{\partial w_{1}}{\partial x}
$$

The small reorientation $\theta_{1}$ of the anisotropic sedimenting fibers leads to a sideways slip $u_{1}=-V_{S} \theta_{1}$, with therefore a sideways flux of fibers $n_{0} u_{1}$. A horizontal divergence in this flux changes the initial variation in the number density

$$
\frac{\partial}{\partial t}\left(n_{1} \cos k x\right)+\frac{\partial}{\partial x}\left(n_{o} u_{1}\right)=0 .
$$

Assembling the above expressions, one finds an instability with a growth rate

$$
0\left(\frac{V_{S}}{l}\left(n_{o} l^{3}\right)^{1 / 2}\right)
$$

where $l$ is the length of the fibers. This explanation simplifies details of the rotation of initially randomly oriented fibers, and as a consequence has all wavelengths equally unstable. The full analysis of Koch \& Shaqfeh (1989) finds the longest waves are most unstable. The observed instability may however depend more on the initial density fluctuations rather than on which grows fastest, particularly because the instability can be expected to become nonlinear by the time that the fibers have fallen through their own length.

\subsection{Clusters and streamers}

In dilute suspensions at low Reynolds numbers (i.e. having typical particle Reynolds number $R e_{p}=V_{S \|} l / \nu \approx 10^{-4}$ and cell Reynolds number $R e_{L}=U_{s \|} L / \nu=$ $\left.\operatorname{Re}_{p}(L / l) \approx 10^{-2}\right)$, the existence of the instability and enhanced sedimentation have been observed in fiber-tracking experiments (Herzhaft et al. 1996, Herzhaft 

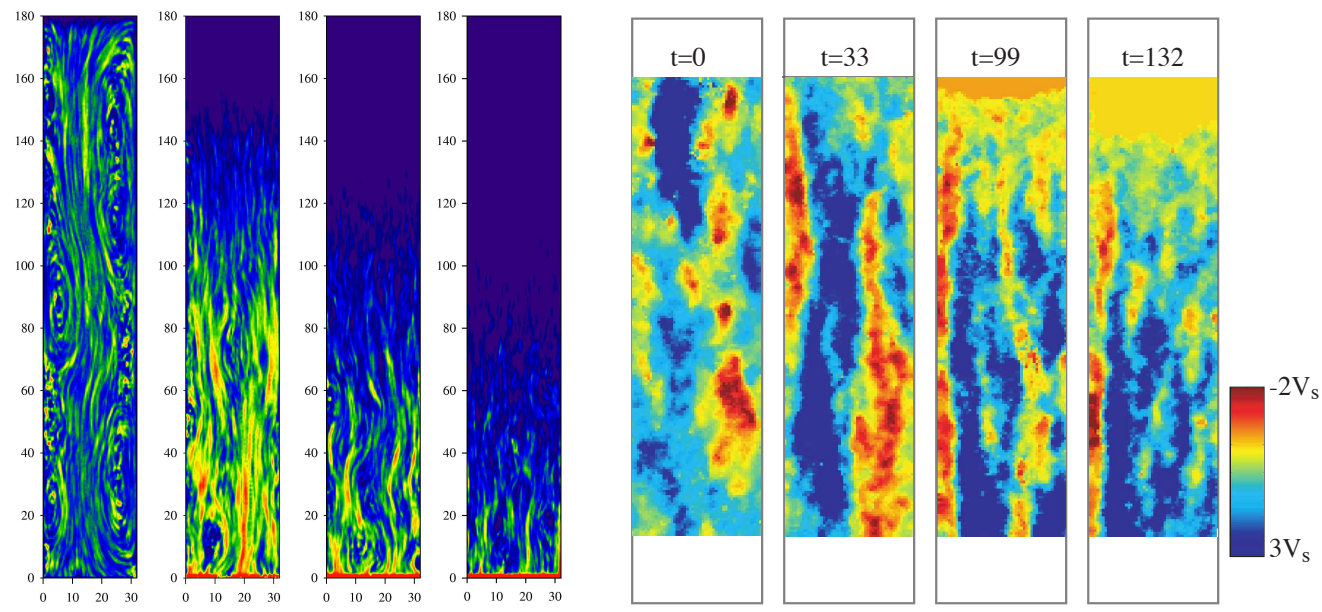

Figure 8: Evolving streamers (left) in simulation with bottom bounding wall [time trace of the concentration field over a short interval in the mid-plane of the box, from left to right $t=0,40,80,120$ (Saintillan et al. 2006a)] and (right) in experiments [vertical component of the velocity field extracted from the median plane of the cell by using PIV (Metzger et al. 2007a)]. The time scale is the Stokes time, i.e. the time for an vertical isolated fiber to sediment half of its length in the experiments and its length in the simulations.

\& Guazzelli 1999). The fibers are observed to orient in the direction of gravity and to form concentrated clusters whose size is of the order of a few fiber lengths and which are evolving objects, constantly dissolving and forming, i.e. capturing and loosing fibers. More recent experiments using PIV measurements (Metzger et al. 2005, Metzger et al. 2007a) have seen the clusters organize into downwards streamers of high density balanced by backflow regions of low density, and the flow structure evolve from long wavelengths to shorter wavelengths, see Figure 8 (right) and Supplemental Movie 3. Similarly, streamers have been observed when bidisperse suspensions sediment (Davis \& Acrivos 1985). While the mean velocity and fiber orientation reach steady states, the flow structure evolves continuously 

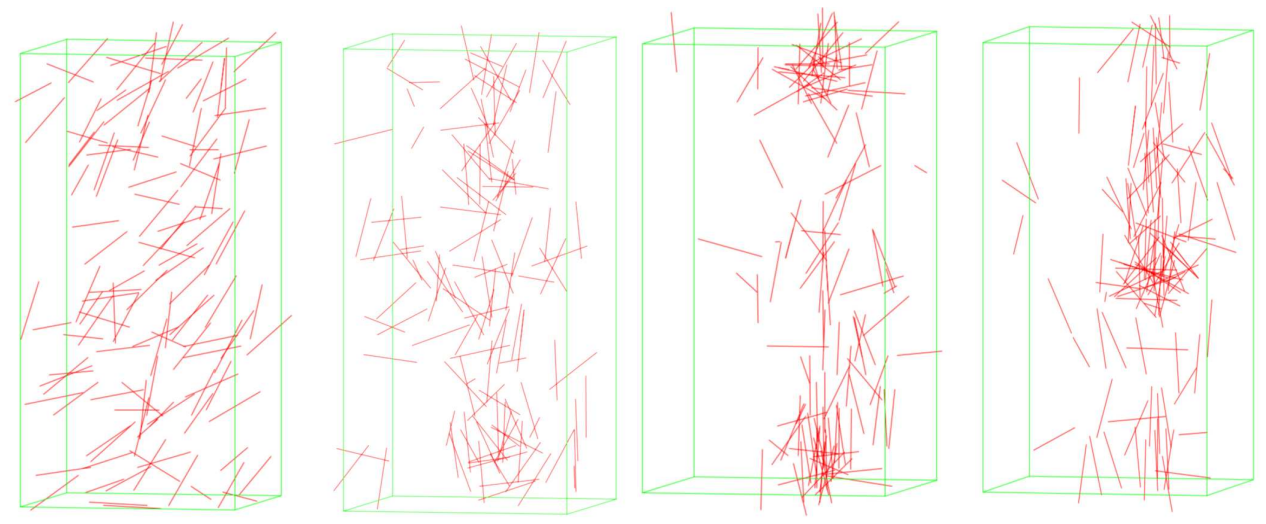

Figure 9: Pictures of the formation and evolution of clusters in a $4 \times 4 \times 8$ periodic box simulation using slender-body dynamics and comprising 128 fibers (Butler \& Shaqfeh 2002): The first (from the left) is the initial condition, the second is around the time when the orientation distribution attains steady state, and the last two are snapshots of the clusters at different times. The time scale is the Stokes time, i.e. the time for an isolated fiber to sediment half of its length.

in time without reaching steady state.

The hydrodynamic instability is also seen in zero-Re numerical simulations of varying degrees of sophistication, ranging from the simplest point-force approximation to the more complex slender-body approximation for the far-field interactions, supplemented by a lubrication approximation for the short-range interactions. When using periodic boundary conditions (Mackaplow \& Shaqfeh 1998, Butler \& Shaqfeh 2002, Saintillan et al. 2005, Gustavsson \& Tornberg 2009), the fibers are observed to clump together and to converge into a single dense streamer which spans the whole height of the periodic box, see Figure 9. The dynamics of clusters within the streamer, i.e. their continual dissolution and formation, show strong similarity with experiments. Realistic predictions are obtained by using boxes elongated in the vertical direction. In particular, (i) the mean 
velocity can reach steady states with values in close agreement with experimental data, (ii) the anisotropy of the fluctuations is $\approx 3$ as in experiments, but the predicted values of the fluctuations are lower, see Figure 7. It is worth mentioning that all the simulations predict a strong vertical alignment of the fibers although with a degree of anisotropy weaker than in the experiments. A small preferred horizontal alignment seen in the experiments (Herzhaft \& Guazzelli 1999, Metzger et al. 2007a) is not captured. A no-flux boundary condition imposed at the bottom of the box has a strong impact on the evolution of the flow structure (Saintillan et al. 2006a). The single large-scale streamer, still seen at initial times, breaks up into smaller streamers in qualitative agreement with the experiments, see Figure 8 and Supplemental Movie 4. The role of the bottom bounding wall may be related to the suppression of the long-wavelength horizontal density fluctuations in suspensions of rigid spheres, see Section 2.2. These simulations capture well the velocity and orientation statistics but the mean velocity did not achieved a steady state, possibly due to the limited size of the simulation.

Some of the features of the instability have been understood but the important issue of the wavelength selection remains unsolved. The linear stability analysis of Koch \& Shaqfeh predicts that the perturbations with the maximum growth rate are those of largest wavelength. In a bounded system, the container size limits the size of the largest possible wavelength. Indeed, the growth of a single streamer spanning the width of the box is reported in the simulations at short times regardless of the boundary conditions used. However, wavelengths scaling with the container dimension are not observed in experiments at early times (Metzger et al. 2007a). Moreover, the streamer structure evolves slowly from long to short wavelengths without reaching a steady state and no simple dependence 
on suspension height, cell cross-section, volume fraction, and properties of the fibers and the fluid is observed. The reduction in wavelength is qualitatively reproduced by simulations using a point-particle method and allowing slip but no penetration at the bottom wall (Saintillan et al. 2006a). However, these simulations suggests that the wavelength selection is controlled by stratification and hydrodynamic diffusion in a scaling similar to that found for the cutoff length by stratification in the suspension of spheres (Saintillan et al. 2006b) but this mechanism does not account for the wavelength reduction in the experiments (Metzger et al. 2007b).

\section{CONCLUDING REMARK AND FUTURE ISSUES}

This review has attempted to present the current state of understanding of the sedimentation of non-Brownian particles at low Reynolds numbers. The difficulties that one encounters lies in the long range nature of the multi-body hydrodynamic interactions between particles. The hydrodynamics are determined by the suspension microstructure, i.e. the orientation and relative position of the particles, which is itself determined by the hydrodynamics. This coupling results in a complex collective dynamics; large fluctuations correlating many thousands of sedimenting spheres and large-scale streamers composed of many sedimenting fibers.

List of summary points:

1. For a monodisperse suspension of sedimenting spheres, there are large fluctuations in the velocities of the particles, often as large as the mean settling speed, and these fluctuations have a strong anisotropy favoring the vertical direction. 
2. The velocity fluctuations have a correlation length of twenty mean interparticle separations, $20 a \phi^{1 / 3}$, a value which seems robust across a wide range of volume fractions. The correlation length is a key to understanding whether or not an experimental container is large or not. We note few numerical simulations had both sufficient particles and sufficient spatial resolution to be considered large. There is currently no theoretical prediction of the value. While a few interparticle separations is a natural size, it is difficult to imagine an argument producing the large $20 a \phi^{1 / 3}$, which imply blobs of 8000 cooperative particles.

3. A weak stratification, i.e. a vertical variation in the concentration of the particles, may be important. Its role at inhibiting velocity fluctuations in the descending front is now well established for very dilute suspensions. In more concentrated suspensions, hindered settling sharpens the front and so stratification can be expected to be less important.

4. Two distinct regimes of sedimentation have been identified for suspensions of fibers. In the dilute regime, the homogenous suspension is unstable and the mean sedimentation velocity can exceed the maximum sedimentation velocity of an isolated vertical fiber. In a semi-dilute regime, the mean velocity is hindered and the hindrance is more severe than for a suspension of spheres.

5. The hydrodynamic instability which occurs in the dilute regime of sedimentation for suspensions of fibers has been demonstrated by experiments, theory, and simulations. The fibers are observed to orient in the direction of gravity and form clusters which assemble into downward streamers balanced by backflow regions of lower density. This cluster and streamer dynamics is 
captured very well by numerical simulations but the wavelength selection is not yet resolved.

While the issues discussed in this review are mostly settled, there remains challenges in systems more complex than spheres and fibers in Newtonian fluids.

List of future issues:

1. Sedimentation of platelets and deformable particles which probably suffers a similar structural instability as fibers (Saintillan et al. 2006a).

2. Sedimentation in a non-Newtonian suspending fluid: One of the most spectacular manifestations observed is the formation of particle rich structures that take the shape of vertical columns in a shear-thinning fluid and that form several conical piles on the bottom (Mora et al. 2005).

3. Influence of finite inertia: Inertia can induce qualitative change to the dynamics and structure of suspension of settling spheres (Hinch 1988, Koch 1993, Yin \& Koch 2007, Yin \& Koch 2008) and to the instability of sedimenting fibers (Kuusela et al. 2003, Shin et al. 2009).

\section{Sidebar highlighting a related topic: Falling clouds}

A cloud of particles sedimenting in a viscous fluid evolves into a torus that becomes unstable and breaks up into secondary droplets which deform into tori themselves in a repeating cascade (Adachi et al. 1978, Machu et al. 2001). This instability occurs even in the complete absence of inertia and without needing to perturb the initial shape (Metzger, Nicolas \& Guazzelli 2007). The particles circulate in closed toroidal streamlines as predicted by a continuum approach where the cloud is modeled as an effective medium of higher density. Fluctua- 
tions arising from the multibody character of the hydrodynamic interactions cause particles to depart from these streamlines and to be carried into a downstream tail (Nitsche \& Batchelor 1997). Because the lost particles are those located in the circulation rim, this depletes the central region and leads to torus formation. The mechanism responsible for the torus further expansion remains unclear, but the breakup can be described as a change in the flow topology that occurs when the torus reaches a critical aspect-ratio. Simulations using a point-particle approach containing the minimal physics of the long-range interactions capture this dynamics. Faster breakup is observed for clouds of fibers due to the self motion of the anisotropic particles (Park et al. 2010). See Supplemental Movies 5 and 6 .

\section{Supplemental movies}

- Movie1: Sedimentation of a suspension of spheres having $\phi \approx 0.3$ in a test tube at low Reynolds number showing a sharp front between the clear fluid and the suspension (Credits: B. Metzger \& É Guazzelli).

- Movie2: Sedimentation of a suspension of fibers having $\phi \approx 0.005$ in a test tube at low Reynolds number showing a diffuse front and the formation of clusters of fibers which organize in downward streamers (Credits: B. Metzger \& É Guazzelli).

- Movie3: Sedimentation of a dilute suspension of fluorescing fibers within a laser sheet showing the evolution of the streamer structure (Credits: B. Metzger, J. E. Butler \& É Guazzelli) (accelerated movie).

- Movie4: (left) Point-fiber simulations with bottom wall (Credits: D. Saintillan, E. Darve \& E. S. G. Shaqfeh) and (right) experiments (Credits: B. Metzger, J. E. Butler \& É Guazzelli) under similar conditions (accelerated 
movie).

\section{Supplemental movies for the sidebar}

- Movie5: Bottom view of a cloud of colored spheres settling in silicon oil (Metzger, Nicolas \& Guazzelli 2007).

- Movie6: Side and bottom view of a simulation of a sedimenting cloud of point particles in an infinite fluid. The initial number of particles is 3000 but only half of them are plotted (Metzger, Nicolas \& Guazzelli 2007). The time scale is the Stokes time of the cloud.

\section{DISCLOSURE STATEMENT}

The authors are not aware of any biases that might be viewed as affecting the objectivity of this review.

\section{ACKNOWLEDGMENTS}

We thank L. Bergougnoux, J. E. Butler, B. Metzger, M. Nicolas, D. Saintillan, P. Snabre for supplying data, pictures, and movies. While writing this, EJH was partially supported by Award KUK-C1-013-04 made by King Abdullah University of Science and Technology (KAUST).

\section{Literature Cited}

Adachi K, Kiriyama S, Koshioka N. 1978. The behaviour of a swarm of particles moving in a viscous fluid. Chem. Engng. Sci. 33:115-121.

Anselmet M-C. 1989 Contribution à l'étude des systèmes fluide-particules : suspensions de cylindres, lits fluidisés. Thèse, Université de Provence. 
Batchelor GK. 1972. Sedimentation in a dilute dispersion of spheres. J. Fluid Mech. 52:245-268.

Bergougnoux L, Ghicini S, Guazzelli É, Hinch EJ. 2003. Spreading fronts and fluctuations in sedimentation. Phys. Fluids 15:1875-1887.

Bergougnoux L., Guazzelli É. 2009. Non-Poisson statistics of settling spheres. Phys. Fluids.

Bławdziewicz J. circa 1995. private communication.

Brenner MP. 1999. Screening mechanisms in sedimentation, Phys. Fluids 11:754772 .

Butler JE, Shaqfeh ESG. 2002. Dynamic simulations of the inhomogeneous sedimentation of rigid fibres. J. Fluid Mech. 468:205-237.

Caflisch RE, Luke JHC. 1985. Variance in the sedimenting speed of a suspension. Phys. Fluids 28:759-760.

Chehata Gómez D, Bergougnoux L, Hinch EJ, Guazzelli É. 2007. On stratification control of the velocity fluctuations in sedimentation," Phys. Fluids 19:098102.

Chehata Gómez D, Bergougnoux L, Guazzelli É, Hinch EJ. 2008. Spreading fronts in sedimentation of dilute suspension of spheres. Phys. Fluids 20:023302.

Chehata Gómez D, Bergougnoux L, Guazzelli É, Hinch EJ. 2009. Fluctuations and stratification in sedimentation of dilute suspensions of spheres. Phys. Fluids 21:093304.

Cowan ML, Page JH, Weitz DA. 2000 Velocity fluctuations in fluidized suspensions probed by ultrasonic correlation spectroscopy Phys. Rev. Letters 85:453456.

Cunha FR, Abade GC, Sousa AJ, Hinch EJ. 2002. Modeling and direct simulation of velocity fluctuations and particle-velocity correlations in sedimentation. $J$. 
Fluids Eng. 124:957-968.

Davis RH. 1996. Hydrodynamic diffusion of suspended particles: a symposium. J. Fluid Mech. 310: 325-335.

Davis RH, Acrivos A. 1985. Sedimentation of noncolloidal particles at low Reynolds numbers. Ann. Rev. Fluid Mech. 17: 91-118.

Davis RH, Hassen MA. 1988. Spreading of the interface at the top of a slightly polydisperse sedimenting suspension. J. Fluid Mech. 196:107-134 with Corrigendum. 1989. J. Fluid Mech. 202:598-599.

Guazzelli É. 2001. Evolution of particle-velocity correlations in sedimentation. Phys. Fluids 13:1537-1540.

Gustavsson K, Tornberg A-K. 2009 Gravity induced sedimentation of slender fibers. Phys. Fluids 21:123301.

Ham JM, Homsy GM. 1988. Hindered settling and hydrodynamic dispersion in quiescent sedimenting suspension. Int. J. Multiphase Flow 14:533-546.

Herzhaft B, Guazzelli É, Mackaplow MB, Shaqfeh ESG. 1996. Experimental investigation of the sedimentation of a dilute fiber suspension. Phys. Rev. Lett. $77: 290-293$

Herzhaft B, Guazzelli É. 1999. Experimental study of the sedimentation of dilute and semi-dilute suspensions of fibres. J. Fluid Mech. 384:133-158.

Hinch EJ. 1977. An averaged-equation approach to particle interactions in a fluid suspension. J. Fluid Mech. 83: 695-720.

Hinch EJ. 1988. Sedimentation of small particles. In Disorder and Mixing, eds E Guyon, J-P Nadal, Y Pomeau, pp. 153-161. Dordrecht: Kluwer Academic.

Koch DL. 1993. Hydrodynamic diffusion in dilute sedimenting suspensions at moderate Reynolds numbers. Phys. Fluids A 5:1141-1155. 
Koch DL. 1994 Hydrodynamic Diffusion in a Suspension of Sedimenting Point Particles with Periodic Boundary Conditions. Phys. Fluids 6:2894-2900.

Koch DL, Shaqfeh ESG. 1989. The instability of a dispersion of sedimenting spheroids. J. Fluid Mech. 209:521-542.

Koch DL, Shaqfeh ESG. 1991. Screening mechanisms in sedimenting suspension. J. Fluid Mech. 224:275-303.

Kumar P, Ramarao BV. 1991. Enhancement of the sedimentation rates in fibrous suspensions. Chem. Engng Commun. 108:381-401.

Kuusela E, Lahtinen JM, Ala-Nissila T. 2003. Collective effects in settling of spheroids under steady-state sedimentation. Phys. Rev. Lett. 90:094502.

Ladd AJC. 1993, Dynamical simulations of sedimenting spheres. Phys. Fluids A $5: 299-310$.

Ladd AJC. 1996. Hydrodynamic Screening in Sedimenting Suspensions of nonBrownian Spheres. Phys. Rev. Lett. 76:1392-1395.

Ladd AJC. 1997 Sedimentation of homogeneous suspensions of non-Brownian spheres. Phys. Fluids 9:491-499.

Ladd AJC. 2002. Effect of container walls on the velocity fluctuations of sedimenting spheres. Phys. Rev. Lett. 88:048301.

Lee S, Yang Y, Choi C, Lee T. 1992. Combined effect of sedimentation velocity fluctuation and self-sharpening on interface broadening. Phys. Fluids 4:26012606.

Lei X, Ackerson BJ, Tong P. 2001.Settling Statistics of Hard Sphere Particles. Phys. Rev. Lett. 86:3300-3303.

Levine A, Ramaswamy S, Frey E, Bruinsma R. 1998. Screened and unscreened phases in sedimenting suspensions. Phys. Rev. Lett. 81:5944-5947. 
Luke JHC. 2000. Decay of velocity fluctuations in a stably stratified suspension. Phys. Fluids 12:1619-1621.

Machu G, Meile W, Nitsche LC, Schaflinger U. 2001. Coalescence, torus formation and breakup of sedimenting drops: Experiments and computer simulations. $J$. Fluid Mech. 447:299-336.

Mackaplow MB, Shaqfeh ESG. 1998. A numerical study of the sedimentation of fibre suspension. J. Fluid Mech. 376:149-182.

Martin J, Rakotomalala N, Salin D. 1994. Hydrodynamic dispersion broadening of sedimenting front. Phys. Fluids 6:3215-3217.

Metzger B, Guazzelli É, Butler JE. 2005. Large-scale streamers in the sedimentation of a dilute fiber suspension. Phys. Rev. Lett. 95:164506.

Metzger B, Butler JE, Guazzelli É. 2007. Experimental investigation of the instability of a sedimenting suspension of fibres. J. Fluid Mech. 575:307332.

Metzger B, Butler JE, Guazzelli É. 2007. On wavelength selection by stratification in the instability of settling fibers. Phys. Fluids 19:098105.

Metzger B, Nicolas M, Guazzelli É. 2007. Falling clouds of particles in viscous fluids. J. Fluid Mech. 580:283-301.

Mora S, Talini L, Allain C. 2005. Structuring Sedimentation in a Shear-Thinning Fluid. Phys. Rev. Lett. 95:088301.

Mucha PJ, Brenner MP. 2003. Diffusivities and front propagation in sedimentation. Phys. Fluids 15:1305-1313.

Mucha PJ, Tee S-Y, Weitz DA, Shraiman BI, Brenner MP. 2004. A model for velocity fluctuations in sedimentation. J. Fluid Mech. 501:71-104.

Nicolai H, Guazzelli E. 1995. Effect of the vessel size on the hydrodynamic diffusion of sedimenting spheres. Phys. Fluids 7:3-5. 
Nicolai H, Herzhaft B, Hinch EJ, Oger L, Guazzelli E. 1995. Particle velocity fluctuations and hydrodynamic self-diffusion of sedimenting non-Brownian spheres. Phys. Fluids 7:12-23.

Nitsche JM, Batchelor GK. 1997. Break-up of a falling drop containing dispersed particles. J. Fluid Mech. 340:161-175.

Nguyen N-Q, Ladd AJC. 2004 Microstructure in a settling suspension of hard spheres. Phys. Rev. Lett. E 69:050401.

Nguyen N-Q, Ladd AJC. 2005 Sedimentation of hard-sphere suspensions at low Reynolds number. J. Fluid Mech. 525:73-104.

Park J, Metzger B, Guazzelli É, Butler JE. 2010. A cloud of rigid fibres sedimenting in a viscous fluid. J. Fluid Mech. in press.

Ramaswamy S. 2001. Issues in the statistical mechanics of steady sedimentation. Advances in Physics 50:297-341.

Saintillan D, Darve E, Shaqfeh ESG. 2005. A smooth particle-mesh Ewald algorithm for Stokes suspension simulations: The sedimentation of fibers. Phys. Fluids 17:033301.

Saintillan D, Shaqfeh ESG, Darve E. 2006a. The growth of concentration fluctuations in dilute dispersions of orientable and deformable particles under sedimentation. J. Fluid Mech. 553:347-388.

Saintillan D, Shaqfeh ESG, Darve E. 2006b. The effect of stratification on the wavenumber selection in the instability of sedimenting spheroids. Phys. Fluids 18:121503.

Segrè PN, Helbolzheimer E, Chaikin PM. 1997. Long-range correlations in sedimentation. Phys. Rev. Lett. 79:2574-2577.

Segré PN, Liu F, Umbanhowar P, Weitz DA. 2001. An effective gravitational 
temperature for sedimentation. Nature 409:594597.

Shin M, Koch DL, Subramanian G. 2009. Structure and dynamics of dilute suspensions of finite-Reynolds-number settling fibers. Phys. Fluids 21:123304.

Snabre P, Pouligny B, Metayer C, Nadal F. 2009. Size segregation and particle velocity fluctuations in settling concentrated suspensions. Rheol. Acta.

Tee S-Y, Mucha PJ, Cipelletti L, Manley S, Brenner MP, Segrè PN, Weitz DA. 2002 Nonuniversal velocity fluctuations of sedimenting particles. Phys. Rev. Lett. 89:054501.

Tee S-Y, Mucha PJ, Brenner MP, Weitz DA. 2007. Velocity fluctuations of initially stratified sedimenting spheres. Phys. Fluids 19:113304.

Tong P, Ackerson BJ. 1998. Analogies between colloidal sedimentation and turbulent convection at high Prandtl numbers. Phys. Rev. E 58:R6931.

Tory EM, Pickard DK. 1986. On the divergence problem in calculating particle velocities in dilute dispersions of identical spheres. Powder Technol. 47:39-42.

Tory EM, Kamel MT, Chan Man Fong, CF. 1992, Sedimentation is container-size dependent. Powder Technol., 73:219-238.

Turney MA, Cheung MK, McCarthy MJ, Powell RL. 1995. Magnetic resonance imaging study of sedimenting suspensions of noncolloidal spheres. Phys. Fluids 7:904-911.

Turney MA, Cheung MK, Powell RL, McCarthy MJ. 1995 Hindered settling of rod-like particles measured with magnetic resonance imaging. AIChE $J$. $41: 251-257$.

Xue J-Z, Herbolzheimer E, Rutgers MA, Russel WB, Chaikin PM. 1992 Diffusion, dispersion, and settling of hard spheres. Phys. Rev. Letters 69:1715-1718.

Yin X, Koch DL. 2007. Hindered settling velocity and microstructure in sus- 
pensions of solid spheres with moderate Reynolds numbers. Phys. Fluids 19:093302.

Yin X, Koch DL. 2008 Velocity fluctuations and hydrodynamic diffusion in finiteReynolds-number sedimenting suspensions. Phys. Fluids 20:043305. 



\section{RECENT REPORTS}

25/09 Preconditioning for active set and projected gradient methods as semi-smooth Newton methods for PDE-constrained optimization

Wathen with control constraints

26/09 Functional differential equations arising in cell-growth

Wake

Begg

27/09 A Cell Growth Model Revisited

Derfel

van Brunt

Wake

28/09 Quasi-steady state reduction of molecular motor-based models of

Newby directed intermittent search

Bressloff

29/09 All-at-once preconditioning in PDE-constrained optimization Rees

Stoll

Wathen

30/09 An hp-Local Discontinuous Galerkin method for Parabolic Integro-Differential Equations

Pani

Yadav

31/09 Stochastic neural field theory and the system-size expansion

Bressloff

32/09 A Hamiltonian Krylov-Schur-type method based on the symplectic Lanczos process

Benner

Faßbender

Stoll

33/09 Nematic liquid crystals : from Maier-Saupe to a continuum theory

Ball

Majumdar

34/09 Tangent unit-vector fields: nonabelian homotopy invariants and the Dirichlet energy

Majumdar

Robbins

Zyskin

35/09 A metabolite-sensitive, thermodynamically-constrained model of

Tran cardiac cross-bridge cycling: Implications for force development during ischemia

Smith

Loiselle

Crampin

36/09 Modelling bacterial behaviour close to a no-slip plane boundary: the influence of bacterial geometry

Shum

Gaffney

Smith

37/09 Optimal L2-error estimates for the semidiscrete Galerkin approx-

Goswami imation to a second order linear parabolic initial and boundary value problem with nonsmooth initial data

Pani

38/09 Optimal L2 estimates for semidiscrete Galerkin methods for parabolic integro-differential equations with nonsmooth data

Goswami

Pani

Yadav 
39/09 Spatially structured oscillations in a two-dimensional excitatory neuronal network with synaptic depression

Kilpatrick

Bressloff

40/09 Stationary bumps in a piecewise smooth neural field model with synaptic depression

Kilpatrick

Bressloff

41/09 Homogenization for advection-diffusion in a perforated domain

Haynes

Hoang

Norris

Zygalakis

42/09 Fast stochastic simulation of biochemical reaction systems by alternative formulations of the Chemical Langevin Equation

Melykuti

Burrage

Zygalakis

43/09 Pseudoreplication invalidates the results of many neuroscientific

Lazic studies

44/09 Cardiac cell modelling: Observations from the heart of the cardiac physiome project

45/09 A Hybrid Radial Basis Function - Pseudospectral Method for Thermal Convection in a 3-D Spherical Shell

Wright

Flyer

46/09 Refining self-propelled particle models for collective behaviour Yates

Baker

Erban

Maini

47/09 Stochastic Partial Differential Equations as priors in ensemble methods for solving inverse problems

Potsepaev

Farmer

Aziz

48/09 DifFUZZY: A fuzzy spectral clustering algorithm for complex data sets

Cominetti et al.

Copies of these, and any other OCCAM reports can be obtained from:

Oxford Centre for Collaborative Applied Mathematics

Mathematical Institute

24 - 29 St Giles'

Oxford

OX1 3LB

England

www.maths.ox.ac.uk/occam 